\title{
Optimization of liquid crystal devices based on weakly conductive layers for lensing and beam steering
}

Jeroen Beeckman, Inge Nys, Oliver Willekens, and Kristiaan Neyts

Citation: J. Appl. Phys. 121, 023106 (2017); doi: 10.1063/1.4973939

View online: http://dx.doi.org/10.1063/1.4973939

View Table of Contents: http://aip.scitation.org/toc/jap/121/2

Published by the American Institute of Physics

\section{Articles you may be interested in}

Photonic crystal properties of self-assembled Archimedean tilings

J. Appl. Phys. 121, 023101023101 (2017); 10.1063/1.4973472

Highly tunable bistability using an external magnetic field in photonic crystals containing graphene and magnetooptical layers

J. Appl. Phys. 121, 023105023105 (2017); 10.1063/1.4973897

Microfluidic metamaterial sensor: Selective trapping and remote sensing of microparticles

J. Appl. Phys. 121, 023102023102 (2017); 10.1063/1.4973492

Analytical equation for the motion picture response time of display devices

J. Appl. Phys. 121, 023108023108 (2017); 10.1063/1.4974006 


\title{
Optimization of liquid crystal devices based on weakly conductive layers for lensing and beam steering
}

\author{
Jeroen Beeckman, ${ }^{\text {a) }}$ Inge Nys, Oliver Willekens, and Kristiaan Neyts \\ Department of Electronics and Information Systems, Ghent University, Ghent, Belgium
}

(Received 23 September 2016; accepted 19 December 2016; published online 12 January 2017)

\begin{abstract}
Liquid crystals are mostly known for their use in displays, but over the past decade these materials have been applied in a number of other devices such as tunable lenses or beam steering devices. A common technique to realize a gradual electric field profile as is required to obtain a gradual refractive index profile in these applications is the use of weakly conductive materials. The weakly conductive layers are able to spread the voltage profile which is applied through well-conductive electrodes at the side of the weakly conductive layer. The simulation and design of such structures is not trivial because two or three dimensional quasi-static electric field profiles need to be calculated. This is due to the fact that the resistivity of the conductive layers and the dielectric properties of the liquid crystal are coupled. An exact solution requires solving a number of coupled differential equations. In this paper, we develop a model to simulate the RC-effects with an approximate model. Published by AIP Publishing. [http://dx.doi.org/10.1063/1.4973939]
\end{abstract}

\section{INTRODUCTION}

Lenses with a tunable focal distance are interesting for a number of applications in which the traditional mechanical focus adaptation is not an option. Quite a number of commercially available tunable focus lenses rely on a system where a fluid is mechanically pushed between flexible substrates $^{1}$ or a system based on electro-wetting. ${ }^{2}$ Another important class of tunable lenses is based on liquid crystals (LCs). ${ }^{3}$ Liquid crystals are electro-optic materials that are widely used in display applications. When applying a voltage over a liquid crystal layer, the average molecular orientation is changed such that it aligns along the electric field vector (for positive dielectric anisotropy liquid crystals). Due to this reorientation, light that is passing through the liquid crystal layer experiences a different refractive index. A suitable positional dependent refractive index profile then leads to a curvature of the phase profile of the light such that the beam is focused (in a tunable lens) or such that the direction of the beam is changed (in beam steering devices). A wide number of approaches have been studied to realize liquid-crystal tunable lenses, using a non-uniform LC layer thickness, ${ }^{4}$ a non-uniform distance to the electrodes, ${ }^{5}$ an optically hidden dielectric structure, ${ }^{6}$ a spatially varying polymer stabilized $\mathrm{LC}^{7}$ or a series of ring-shaped electrodes. ${ }^{8-10}$ Another important class of LC tunable lenses is based on the use of weakly conductive layers. Due to the combined effect of the resistivity of the layer and the capacitance of the LC layer, the uniform weakly conductive layer leads to a spatially varying effective voltage profile. Such a weakly conducting layer approach can be used for tunable lens applications ${ }^{1-14}$ but also for beam steering devices. ${ }^{15,16}$

The sheet resistance of weakly conductive layers is an important parameter in the design of several types of devices. It is well known that large area Organic Light Emitting

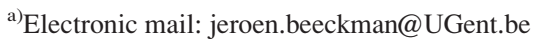

Diode devices (OLEDs) for lighting applications may suffer from a drop in light emission due to the finite sheet conductivity. An intelligent design of conductor grids is necessary. ${ }^{17,18}$ Similar effects are playing a role in solar cells in which the generated current needs to be collected by the electrode grid in an efficient way. ${ }^{19}$ In OLEDs and solar cells, the currents are stationary and the voltage drop is purely related to a resistive effect. In LC devices, the LC layer acts as a capacitor and a possible voltage drop due to a finite sheet conductivity is strongly dependent on the frequency of applied voltage. AC voltages are necessary in LC devices as DC voltages result in unwanted movement of small concentration of ions in the LC material. ${ }^{20}$

In one-dimensional beam steering devices, it is desired to have a linear refractive index profile along one direction. Approximately, this means that also the voltage variation from one contacting electrode to the other needs to be linear. This can be accomplished by using a conductive layer with low sheet resistance. Such a low sheet resistance inevitably leads to a large current. In large-area beam steering devices, this leads to an unacceptably high power consumption. When the sheet resistance of the weakly conductive layer is high, the power consumption is low, but this will lead to a voltage drop between the contacting electrodes. It is thus vital for the proper functioning of the device that the correct sheet resistance of the weakly conductive layer is chosen. Finding the right sheet resistance is not evident as the simulation of such a structure requires the solution of two sets of differential equations in 2D or 3D. The first set of equations is related to the electric field distribution in the structure consisting of perfect conductors, weakly conductive layers, and the anisotropic dielectric LC material. The other set of differential equations relates to the response of the liquid crystal to the applied electric field. From the behavior of the liquid crystal, it is then possible to calculate the optical field propagation through the layer. In this work, we develop an approximate model to simulate the behavior of nematic LC devices 
with weakly conductive layers and the model will be applied to one and two-dimensional beam steering devices and tunable lenses with circular geometry.

\section{THEORETICAL MODEL}

A LC device with weakly conducting layers consists of a LC layer with a uniform thickness as depicted in Fig. 1. The LC layer is not conductive, but in practice the LC needs to be driven with low frequency AC electric fields (e.g., $1 \mathrm{kHz}$ ) to avoid ion drift. ${ }^{20}$ The permittivity of the LC is anisotropic and is given by the $3 \times 3$ tensor $\overline{\bar{\varepsilon}}$. The elements of the tensor depend on the orientation of the average molecular orientation (i.e., the director, given by the vector $\bar{n}$ ) according to the equation

$$
\varepsilon_{i j}=\varepsilon_{\perp} \delta_{i j}+\Delta \varepsilon n_{i} n_{j}
$$

with $\varepsilon_{\perp}$ the permittivity perpendicular to the director and $\Delta \varepsilon=\varepsilon_{\|}-\varepsilon_{\perp}$ the dielectric anisotropy for low frequency electric fields. For the calculation of the LC director, different models exist, either based on a fixed order $^{21}$ or variable order approach. ${ }^{22}$ We will not elaborate the equations describing the director distribution based on the electric field distribution and boundary conditions in the current manuscript, but we refer to the aforementioned references or a textbook on this topic. ${ }^{23}$

Solving the electrical problem can be greatly simplified when assuming that the lateral dimensions are much larger than the thickness $d$ of the LC layer, which in practical realizations is often the case. The typical thickness of the LC layer is in the order of a few $\mu \mathrm{m}$ up to maximum a few tens of $\mu \mathrm{m}$, while the lateral dimensions range from tens of $\mu \mathrm{m}^{16}$ to several millimeter. ${ }^{13}$ With this approximation, we need to find an effective permittivity of the liquid crystal layer which is position dependent: $\varepsilon_{\text {eff }}(x, y)$. This effective permittivity can be calculated by stating that the $z$-component of the dielectric displacement should be constant along the thickness of the LC layer for every $(x, y)$-position: $D_{z}=\varepsilon_{z z}(z) E_{z}(z)$. Using the latter equation and the fact that $V_{1}-V_{2}=\int_{0}^{d} E_{z} d z$ we find the following equation for $\varepsilon_{\text {eff }}$ from $\overline{\bar{\varepsilon}}$ :

$$
\varepsilon_{\mathrm{eff}}=\frac{d}{\int_{0}^{d} \frac{1}{\varepsilon_{z z}} d z} .
$$

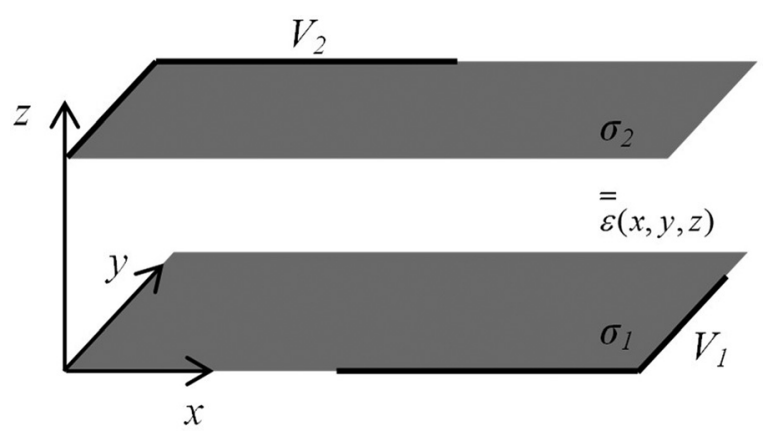

FIG. 1. Sample geometry showing the liquid crystal layer which is sandwiched between two weakly conductive layers. Solid dark lines indicate perfectly conductive electrodes.
In this approximation, only the $\varepsilon_{\mathrm{zz}}$ component is needed. In the case that the LC is planarly aligned and no twist is present, then the director can be described by the tilt angle $\theta$ of the director and the equations reduce to ${ }^{24}$

$$
\begin{aligned}
& \left(K_{11} \cos ^{2} \theta+K_{33} \sin ^{2} \theta\right) \frac{\partial^{2} \theta}{\partial z^{2}}+\frac{1}{2}\left(K_{33}-K_{11}\right) \sin 2 \theta\left(\frac{\partial \theta}{\partial z}\right)^{2} \\
& +\frac{1}{2} \varepsilon_{0} \Delta \varepsilon^{\mathrm{s}} \sin 2 \theta\left|E_{z}\right|^{2}=0
\end{aligned}
$$

In this equation, $K_{11}$ and $K_{33}$ are the elastic constants of the LC for splay and bend. The tilt angle $\theta$ is the angle of the director with the $x y$ plane such that $\bar{n}=\cos (\theta) \overline{1}_{y}+\sin (\theta) \overline{1}_{z}$. Remark that the liquid crystal only responds to the square of the electric field. In most practical LC devices, a sinusoidally varying field is used and for $\left|E_{z}\right|$ the rms value can be used. The resulting $\varepsilon_{\text {eff }}$ for a given voltage can be found in Fig. 2 . For this calculation, the values for the liquid crystal E7 (Merck) are used with $\varepsilon_{\perp}=5.1 \varepsilon_{0}, \varepsilon_{\|}=19.6 \varepsilon_{0}, K_{11}=12 \mathrm{pN}$, $K_{33}=19.5 \mathrm{pN}$ and a fixed tilt angle at the top and bottom substrates of $2^{\circ}$.

The surface current densities $\bar{H}_{1}$ and $\bar{H}_{2}$ at the bottom and top plates, respectively, are given by

$$
\begin{aligned}
& \bar{H}_{1}=-\sigma_{1} d_{1} \nabla V_{1}, \\
& \bar{H}_{2}=-\sigma_{2} d_{2} \nabla V_{2} .
\end{aligned}
$$

In these equations, $\sigma_{i}$ denotes the conductivity and $d_{i}$ is the thickness of the weakly conductive layer, while $V_{i}$ is the potential distribution in the weakly conductive layer. The nabla operator only involves derivatives to $x$ and $y$. Due to the capacity of the LC layer, at each point we can state that the current density $J_{z}$ going from the bottom to the top plate is given by

$$
J_{z}=j \omega \frac{\varepsilon_{\mathrm{eff}}}{d}\left(V_{2}-V_{1}\right)
$$

From now on we assume that all quantities are sinusoidal with an angular frequency $\omega$. The conservation law of charge then leads to

$$
\begin{gathered}
\nabla \cdot \bar{H}_{1}=-J_{z}, \\
\nabla \cdot \bar{H}_{2}=J_{z} .
\end{gathered}
$$

Combining the above equations leads to the two coupled differential equations

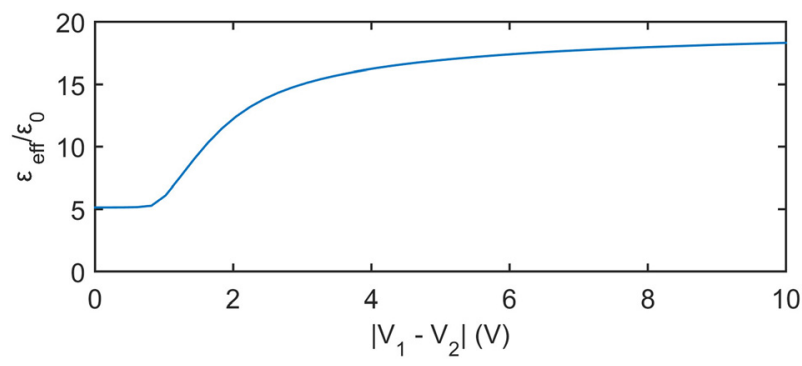

FIG. 2. The variation of $\varepsilon_{\mathrm{eff}}$ as a function of the applied voltage. 


$$
\begin{aligned}
\nabla^{2} V_{1} & =\frac{j \omega \varepsilon_{\mathrm{eff}}}{\sigma_{1} d_{1} d}\left(V_{1}-V_{2}\right), \\
\nabla^{2} V_{2} & =\frac{j \omega \varepsilon_{\mathrm{eff}}}{\sigma_{2} d_{2} d}\left(V_{2}-V_{1}\right) .
\end{aligned}
$$

$\varepsilon_{\text {eff }}$ is a function of $\left|V_{1}-V_{2}\right|$ resulting in a system of nonlinear partial differential equations to be solved.

\section{TWO-DIMENSIONAL GEOMETRY WITH ONE WEAK CONDUCTOR}

In the two-dimensional case, we assume that there is no variation along $y$, while there are two perfect contacts at $x=0$ and $x=L$ on the bottom contact. The above equations simplify greatly if the top conductor has a large conductivity $\left(\sigma_{2} d_{2} \gg \omega \varepsilon_{\max } / d\right)$, which leads to a homogeneous voltage distribution in the top conductor. For simplicity, we set $V_{2}=0$. The equation to be solved reduces to

$$
\nabla^{2} V_{1}=\frac{j \omega \varepsilon_{\mathrm{eff}}}{\sigma_{1} d_{1} d} V_{1}
$$

In a two-dimensional geometry, this equation reduces to a one-dimensional equation with only dependency on $x$

$$
\frac{\partial^{2} V_{1}}{\partial x^{2}}=\frac{j \omega \varepsilon_{\text {eff }}}{\sigma_{1} d_{1} d} V_{1}=j \gamma^{2} V_{1} .
$$

\section{A. Constant permittivity approximation}

If we assume that $\varepsilon_{\mathrm{eff}}$ is position independent, the general solution of this equation is given by

$$
V_{1}=A \exp \left(-\frac{1+j}{\sqrt{2}} \gamma x\right)+B \exp \left(\frac{1+j}{\sqrt{2}} \gamma x\right)
$$

The RC-effect is fully taken into account by the factor $\gamma$. One can define a characteristic distance $x^{c}=1 / \gamma$, which expresses the distance over which the effect takes place. For the ease of notation, we set $\frac{1+j}{\sqrt{2}} \gamma=\alpha$ and we call the distance between the left and right electrode $L$. Consider the case where the left electrode is set to $V_{+}$and the right electrode to $V_{-}$, then the solution is given by

$$
\begin{aligned}
V_{1}(x)= & \frac{V_{+} \exp (\alpha L)-V_{-}}{2 \sinh (\alpha L)} \exp (-\alpha x) \\
& +\frac{V_{-}-V_{+} \exp (-\alpha L)}{2 \sinh (\alpha L)} \exp (\alpha x) .
\end{aligned}
$$

To demonstrate the result of this equation we choose to immediately select realistic values for the different parameters in order to show the effect on the voltage distribution. For the geometry we choose a realistic spacing of $L=50 \mu \mathrm{m}$ between two perfectly conducting electrodes at the bottom. The rest of the material properties are chosen such that the characteristic distance $x^{c}$ equals 10,20 , and $50 \mu \mathrm{m}$. Figures 3 and 4 show the absolute value $|V|$ and the phase difference with the applied voltage $\phi$ with $V_{1}=|V| \exp (j \phi)$ for a distance between the two contacting electrodes of $50 \mu \mathrm{m}$.
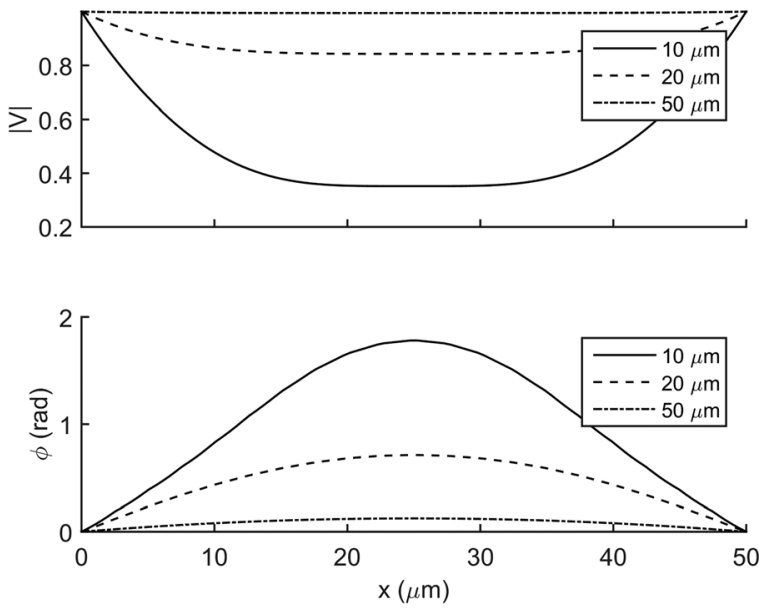

FIG. 3. Absolute value and phase of the voltage signal for a device with a bottom weakly conductive electrode and a top perfect conductor. The bottom conductive layer is contacted with $1 \mathrm{~V}$ at both sides. The different curves correspond to different characteristic distances as denoted in the legend.

Figure 3 shows the situation when the contacting electrodes at the left and the right side are set to $1 \mathrm{~V}$. For large values of the characteristic distance, the absolute value is nearly constant and equal to $1 \mathrm{~V}$ in the gap between the two contacting electrodes. For smaller values of the characteristic distance, there is a considerable voltage drop between the two contacting electrodes due to the RC effects. From the phase plot, it is clear that the voltage signal in the middle is trailing the signals applied to the side. The phase difference in the middle is becoming larger when the characteristic length is decreasing.

A similar observation can be made when $1 \mathrm{~V}$ is applied on the left side and $0 \mathrm{~V}$ on the right side. Instead of a linear voltage variation from left to right, due to the RC effects the voltage is smaller than the linear slope. The voltage trailing seems to keep increasing with increasing $x$. This is due to the fact that the applied voltage is zero at the right side and thus the phase is undefined.
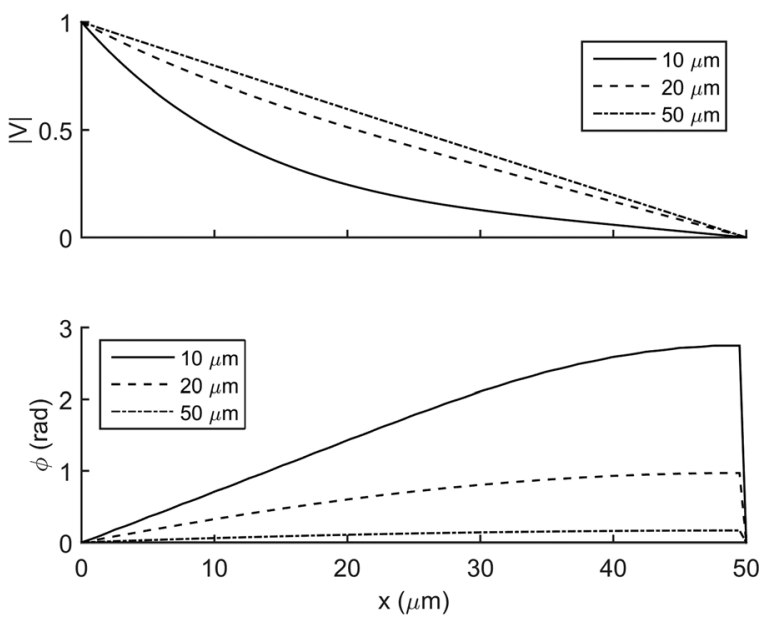

FIG. 4. Absolute value and phase of the voltage signal for a device with a bottom weakly conductive electrode and a top perfect conductor. The bottom conductive layer is contacted with $1 \mathrm{~V}$ at the left and $0 \mathrm{~V}$ at the right. The different curves correspond to different characteristic distances as denoted in the legend. 


\section{B. Variable permittivity}

For some LC materials, there is a big difference between the $\varepsilon_{\perp}$ and the $\varepsilon_{\|}$values. When the characteristic distance is close to the distance between the contacting electrodes, the variation of the effective permittivity can have a big influence on the obtained voltage signal. In contrast to Sec. III A, the voltage profile is also depending strongly on the value of the applied voltage since for small voltages there is almost no variation of the effective permittivity. But for voltages around the threshold, there is a large variation. The calculation involves solving the equations for both the LC (3), the effective permittivity (2), and the potential distribution (12). The partial differential equations are numerically solved using the partial differential equation (PDE) Toolbox in Matlab using finite element discretization. The nonlinearity due to the variable permittivity is taken into account by solving the equations iteratively. After a limited number of iterations, a self-consistent solution is found. Figure 5 shows the results of the calculation for a configuration similar to Fig. 3 . The parameter $\gamma$ is now position dependent and can be written as $\gamma^{2}=\frac{\varepsilon_{\text {eff }}}{\varepsilon_{\perp}} \gamma_{0}^{2}$ with $1 / \gamma_{0}=20 \mu \mathrm{m}$.

\section{Power consumption}

The complex electrical power supplied by an electrode can be calculated by using the following equation at the border of every contacting electrode $i: P=\sum_{i} V_{i} \times I_{i}$ at position $x=x_{i}$, with for a one-dimensional electrode $I_{i}=\mp \sigma d_{1} D$ $\left.\frac{\partial V_{i}}{\partial x}\right|_{x=x_{i}}$. In this equation, $D$ is the length of the electrodes in the invariant direction. The minus and plus signs are, respectively, for an electrode at the left and right edge.

For the case of the constant permittivity approximation and $V_{-}=0$, we find

$$
P=\frac{\sigma d_{1} D V_{+}^{2}}{\tanh \left(\frac{1+j}{\sqrt{2}} \gamma L\right)} \frac{1+j}{\sqrt{2}} \gamma .
$$

The power that we find is a complex number which means that there is both an active and a reactive power
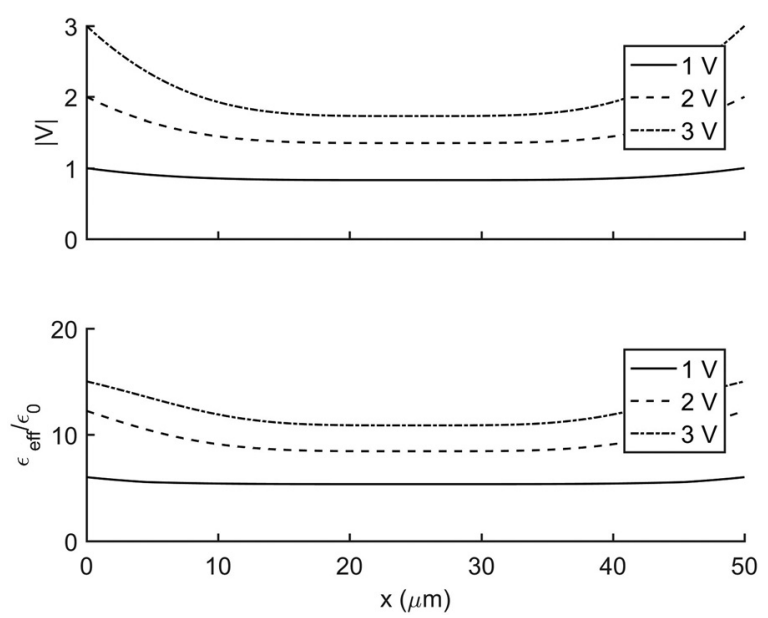

FIG. 5. Absolute value of the voltage signal and the variation of the effective permittivity for a device with a bottom weakly conductive electrode and a top perfect conductor. The bottom conductive layer is contacted with, respectively, 1,2 , and $3 \mathrm{~V}$ on both sides. component. Since most sources are not able to compensate for the reactive power, it is fairly correct to state that the power consumption is equal to the absolute value of $P$. If $\gamma$ is much smaller than 1 (meaning, high conductivity of the layer) then the voltage profile is changing in a linear way from left to right and Equation (15) results in a real number (only active power). Then the power is equal to

$$
P=\frac{\sigma d_{1} D V_{+}^{2}}{L} .
$$

To use the device for large area beam steering, it is necessary to repeat the structure multiple times. Then we obtain a configuration at the lower plane as illustrated in Fig. 6. The left side of the weakly conductive layer is connected through the bottom electrode on the figure, while the right side is connected through the top one.

For this simple geometry, assuming an active area of $D$ by $L_{\text {tot }}$ the total power consumption of the device equals

$$
P=\frac{\sigma d_{1} D V_{+}^{2} L_{\mathrm{tot}}}{L^{2}} .
$$

Alternatively, Equation (15) should be multiplied by $L_{\text {tot }} / L$ in the case when the sheet conductivity is not high. Using realistic values for a LC beam steerer of $1 \mathrm{~cm}^{2}$ surface area $\left(d=10 \mu \mathrm{m}, \omega=2 \pi \times 1 \mathrm{kHz}, \varepsilon_{\mathrm{eff}}=10 \times \varepsilon_{0}\right.$, $L=50 \mu \mathrm{m}$ ) we plot the power in Fig. 7. For large values of the sheet conductivity, the resistive power prevails and the total power $|P|$ is equal to the active power given by Equation (17). For low values of the sheet conductivity, the potential drops to zero quickly when moving away from the left electrode. The power consists of equal parts of resistive and capacitive losses. As was made clear in the previous paragraph, the optimal value of the characteristic distance should be comparable to the distance between the two contacting electrodes $L$. For these values of the characteristic distance we are in the region of the graph where the active power is coming close to the total power. This means that for optimal values of the sheet conductivity (around $10^{-10} \mathrm{~S}$ ) the beam steerer of $1 \mathrm{~cm}^{2}$ total active area results in a power consumption of a few $\mu \mathrm{W}$, which is very reasonable. Such

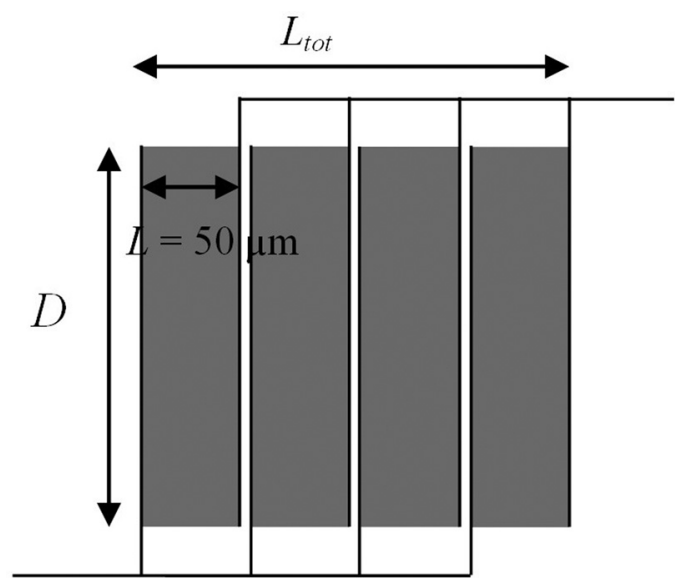

FIG. 6. Geometry of a one-dimensional steerer for calculating the electrical power consumption. 


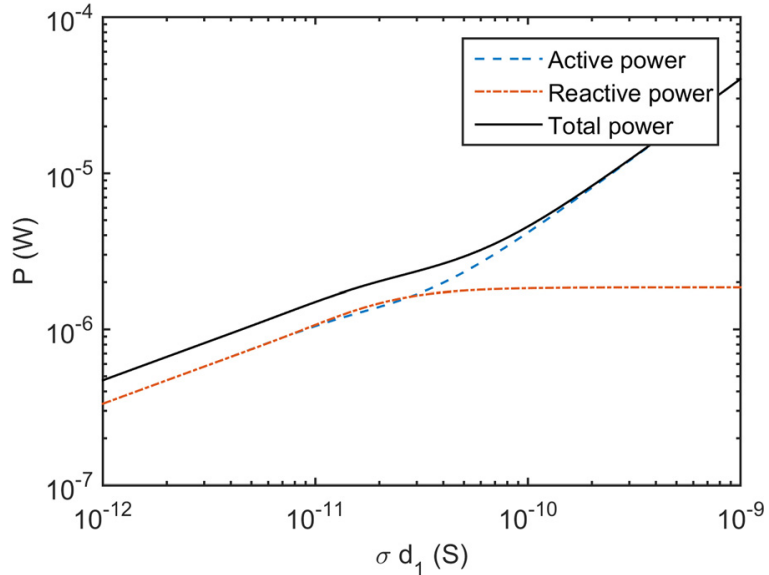

FIG. 7. Power consumption for a $1 \mathrm{~cm}^{2}$ beam steerer as a function of the sheet conductivity of the weakly conductive layer.

values of the sheet conductivity can be obtained with conductive polymers, such as poly (3, 4-ethylenedioxythiophene) polystyrene sulfonate (PEDOT:PSS). In the work of Shang et al. ${ }^{16}$ for example, conductive polymer layers with a sheet resistance of $11 \mathrm{G} \Omega / \mathrm{sq}$ are used, equal to a sheet conductivity of about $10^{-10} \mathrm{~S}$.

\section{THREE-DIMENSIONAL GEOMETRIES}

\section{A. Circular geometry}

Configurations which are circularly symmetric can be solved analytically in the case of only one weak conductor layer and constant permittivity. Rewriting Equation (12) in polar coordinates leads to

$$
\frac{\partial^{2} V_{1}}{\partial r^{2}}+\frac{1}{r} \frac{\partial V_{1}}{\partial r}+\frac{1}{r^{2}} \frac{\partial^{2} V_{1}}{\partial \phi^{2}}-j \gamma^{2} V_{1}=0
$$

Separation of variables $V_{1}(r, \phi)=R(r) \Phi(\phi)$ and expressing that there is no variation in $\phi$ lead to the equation

$$
r^{2} \frac{d^{2} R}{d r^{2}}+r \frac{d R}{d r}-j \gamma^{2} r^{2} R=0
$$

of which the solution is the zeroth order Bessel function

$$
R(r) \sim J_{0}\left(\frac{1+j}{\sqrt{2}} \gamma r\right) .
$$

\section{B. Resistive lens with an outer contact ring}

An obvious example of a three-dimensional geometry is a lens with a tunable focal length. Such a lens consists of a weakly conductive circular area which is contacted with a circular outer ring of a highly conductive material on one substrate. In order to achieve lensing, the resulting phase profile of light propagating through the structure needs to be a parabolic profile. For a convex lens with a positive diopter, the optical path length (OPL or refractive index) in the middle area needs to be larger than the outer regions. This means that the voltage over the planarly aligned liquid crystal needs to be larger at the edge compared to the center. Setting the boundary condition $V(R, \phi)=V_{0}$ leads to the following analytical solution in the constant permittivity approximation

$$
V_{1}(R, \phi)=V_{0} \frac{J_{0}\left(\frac{1+j}{\sqrt{2}} \gamma r\right)}{J_{0}\left(\frac{1+j}{\sqrt{2}} \gamma R\right)} .
$$

The absolute value of the resulting $V_{1}$ along the diameter of the lens is shown in Fig. 8. In order to achieve maximum focusing, the voltage near the center area should be close to zero. As opposed to Sec. III, the ideal conductivity in this case (neglecting electrical power consumption) should not be high in order to obtain the ideal potential profile. A highly conductive layer results in a nearly constant potential profile, which is obviously unwanted.

In the limit of high conductivity $\gamma R \ll 1$, the Bessel function can be approximated by the first terms of its series expansion and $V_{1}$ takes the form

$$
\begin{aligned}
\frac{V_{1}(R, \phi)}{V_{0}} \approx & {\left[J_{0}\left(\frac{1+j}{\sqrt{2}} \gamma R\right)\right]^{-1} } \\
& \times\left[1-\left(\frac{1+j}{\sqrt{2}} \frac{\gamma r}{2}\right)^{2}+\frac{1}{4}\left(\frac{1+j}{\sqrt{2}} \frac{\gamma r}{2}\right)^{4}\right] .
\end{aligned}
$$

In the first order, the voltage profile as a function of $r$ is parabolic. If the LC would respond linearly to applied voltage in terms of refractive index profile, this would result in the desired parabolic refractive index profile of an ideal lens. Unfortunately, in reality, a number of nonlinear effects come into play which makes the profile deviate from the parabolic one as will be shown and explained in Subsection IV D.

\section{Power consumption}

Similar to that in Section IIIC, we can find the power consumption of the device

$$
\begin{aligned}
P & =V_{0} \sigma_{1} d_{1} 2 \pi R \frac{\partial}{\partial r}\left[V_{1}(r, \phi)\right]_{r=R} \\
& =-V_{0}^{2} \sigma_{1} d_{1} 2 \pi \frac{1+j}{\sqrt{2}} \gamma R \frac{J_{1}\left(\frac{1+j}{\sqrt{2}} \gamma R\right)}{J_{0}\left(\frac{1+j}{\sqrt{2}} \gamma R\right)} .
\end{aligned}
$$

Note that in the latter equation $\gamma$ depends on $\sigma_{1} d_{1}$. We can rewrite the equation as

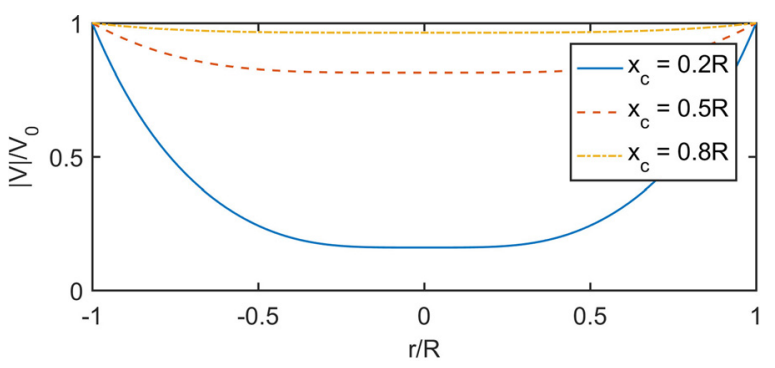

FIG. 8. Absolute value of the voltage along the diameter of a ring-shaped perfect conductor with radius $R$ for different values of $x_{c}$. 


$$
P=-V_{0}^{2} \frac{\omega \varepsilon_{\mathrm{eff}}}{\gamma^{2} d} 2 \pi \frac{1+j}{\sqrt{2}} \gamma R \frac{J_{1}\left(\frac{1+j}{\sqrt{2}} \gamma R\right)}{J_{0}\left(\frac{1+j}{\sqrt{2}} \gamma R\right)} .
$$

Suppose that we are interested to know the power consumption as a function of the sheet conductivity $\sigma_{1} d_{1}$ for a fixed radius $R$ of the circular lens and fixed $\omega$, we can plot $P$ as a function of $x_{c}=1 / \gamma$. The result is shown in Fig. 9 .

In contrast to the power consumption of the twodimensional geometry shown in Fig. 7, the power does not go to infinity when $\sigma d_{1}$ increases (or alternatively when $x_{c}$ increases) because the outer ring is a single electrode and no current can flow towards another electrode. Instead, the power reaches a finite value for large values of $\sigma_{1} d_{1}$ and is completely imaginary. For values which are acceptable for tunable lenses, $x_{c}$ in the range of $0.2 R \rightarrow 0.5 R$ (see Fig. 8), the power is again a mixture of active and reactive power.

\section{Numerical simulations of a tunable lens}

A lens with good optical quality should have a parabolic Optical Path Length (OPL) variation along the radius of the lens. The voltage profiles in Fig. 8 look more or less parabolic, but this is not sufficient because the response of the LC as a function of the applied voltage is very nonlinear. The optical path length can be calculated using the equation for the refractive index as a function of the tilt angle of the LC director

$$
n_{\mathrm{eff}}=\frac{n_{\|} n_{\perp}}{\sqrt{n_{\|}^{2} \sin ^{2}(\theta)+n_{\perp}^{2} \cos ^{2}(\theta)}} .
$$

The $n_{\text {eff }}$ needs to be integrated over the thickness of the LC layer to obtain the OPL. Dividing the OPL by the LC layer thickness results in an average refractive index as shown in Fig. 10. A circular area of $50 \mu \mathrm{m}$ diameter with an outer ring set to voltages ranging from 1 to $15 \mathrm{~V}$ is assumed. The characteristic distance $x_{c}$ is chosen such that the voltage in the center is close to zero, i.e., in the order of a few hundred millivolt. From our simulations, it is found that characteristic distances around $10 \mu \mathrm{m}(=0.4 R)$ result in refractive index profiles which combine an acceptable compromise between tuning range of the focal distance and lens distortions (i.e., deviation from the parabolic profile).

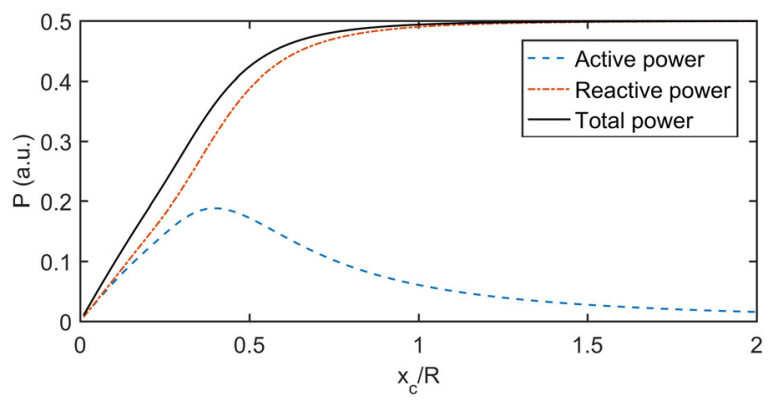

FIG. 9. Power consumption of a circular lens with the outer contact ring as a function of the characteristic distance divided by the lens radius.
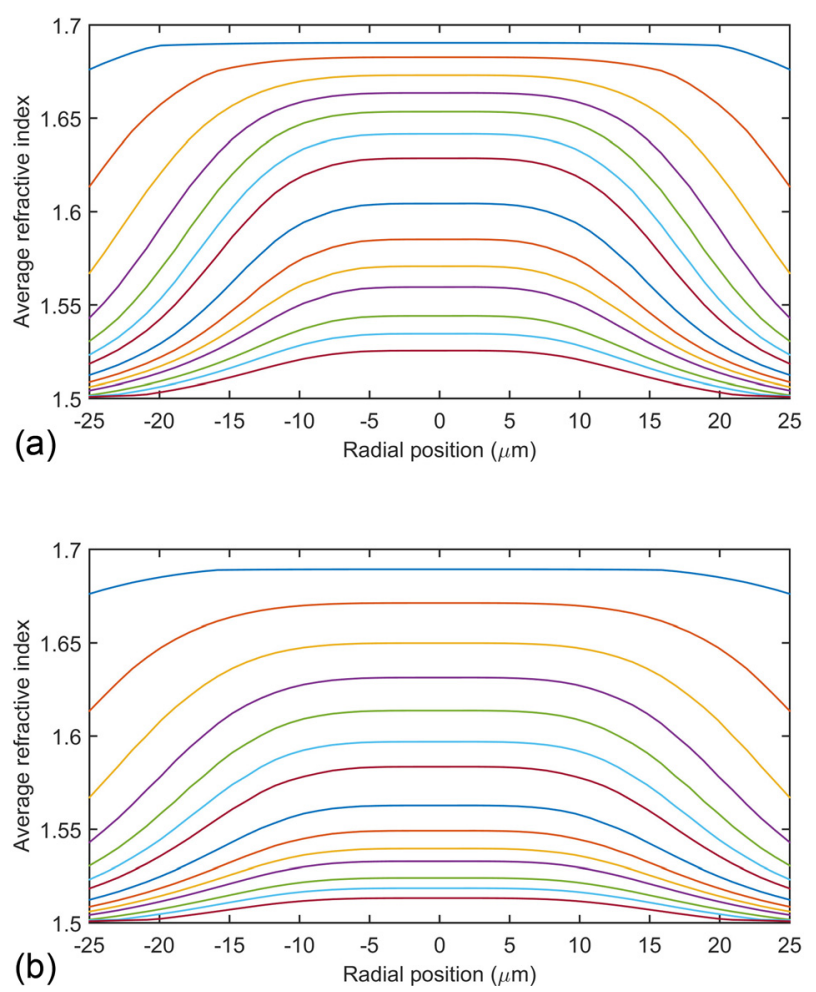

FIG. 10. Refractive index profile inside a ring-shaped PEC with a weakly conductive layer of $x_{c}=10 \mu \mathrm{m}$ (top) and $x_{c}=12 \mu \mathrm{m}$ (bottom). The different curves are for voltages on the PEC ring ranging from 1 to $15 \mathrm{~V}$.

The refractive index profiles in Fig. 10 can be further analyzed. Although the theoretical model predicts a parabolic variation of the voltage (in first order approximation, see Eq. (22)), the OPL profiles in Fig. 10 deviate strongly from the ideal parabolic profile. This is due to two reasons:
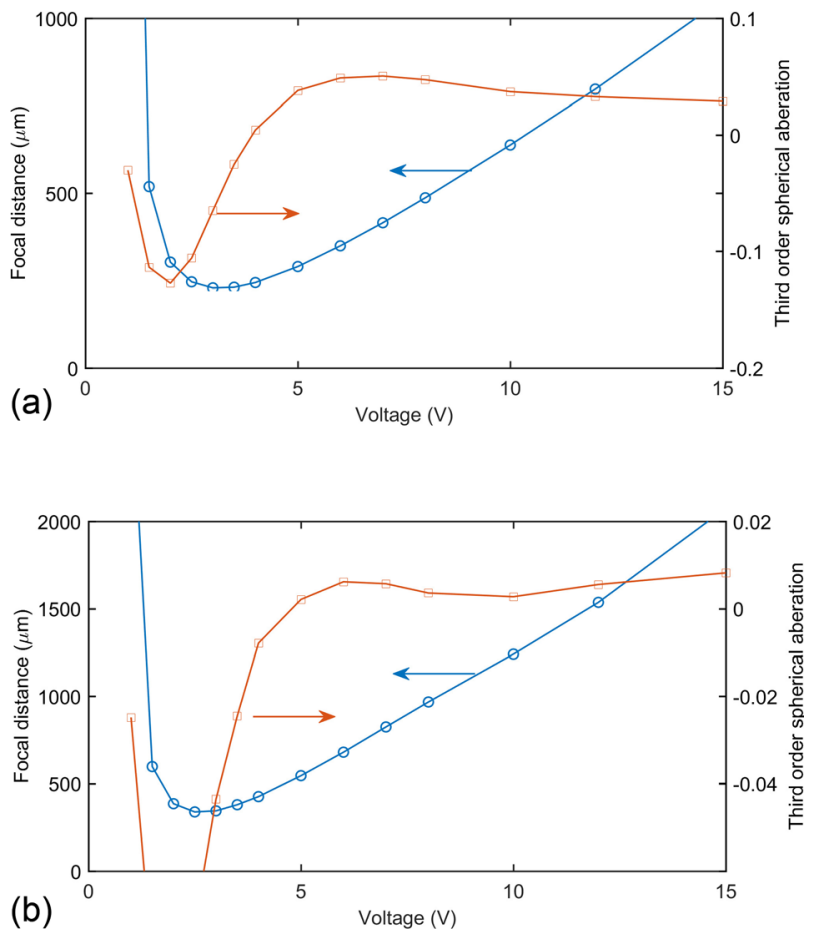

FIG. 11. Analysis of the configuration of Fig. 10 in terms of focal distance and lens aberrations. $x_{c}=10 \mu \mathrm{m}$ (top) and $x_{c}=12 \mu \mathrm{m}$ (bottom). 


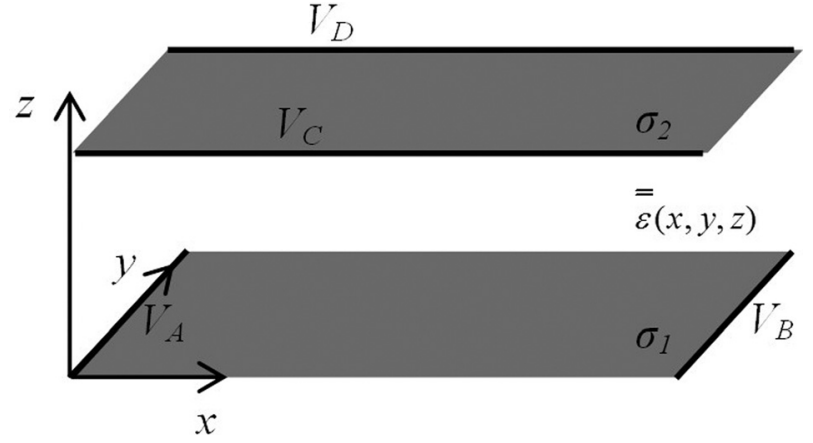

FIG. 12. Configuration for two-dimensional steering. first, the voltage profile is not parabolic due to the combined effect of a voltage dependent permittivity and the fact that the first order approximation in Eq. (22) is not always valid. Second, the OPL as a function of applied voltage is strongly nonlinear. A different LC mode which shows a more linear response as a function of voltage would result in more ideal profiles. The focal distance of the main focal point and the lens aberration is calculated by fitting the first two (circularly symmetric) Zernike polynomials $Z_{2}^{0}(\rho)=a_{3}\left(2 \rho^{2}-1\right)$ and $Z_{4}^{0}(\rho)=a_{8}\left(6 \rho^{4}-6 \rho^{2}+1\right)$ with the OPL variation as a function of $\rho=r / R{ }^{25}$ An LC layer thickness of $10 \mu \mathrm{m}$ is assumed. $Z_{4}^{0}$ is often called the third order spherical aberration, and is a good measure for the deviation from the ideal (a)

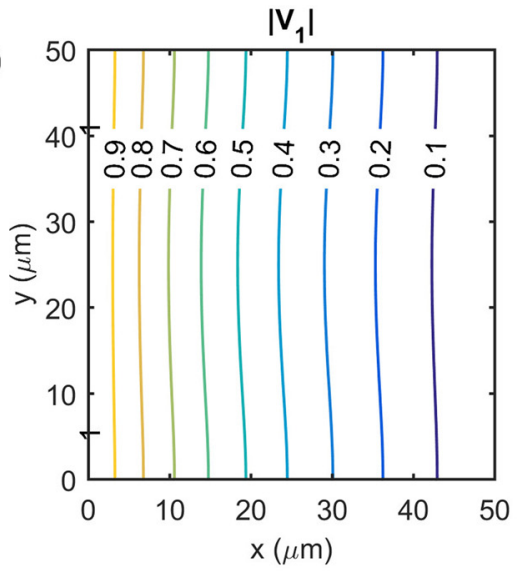

(b)

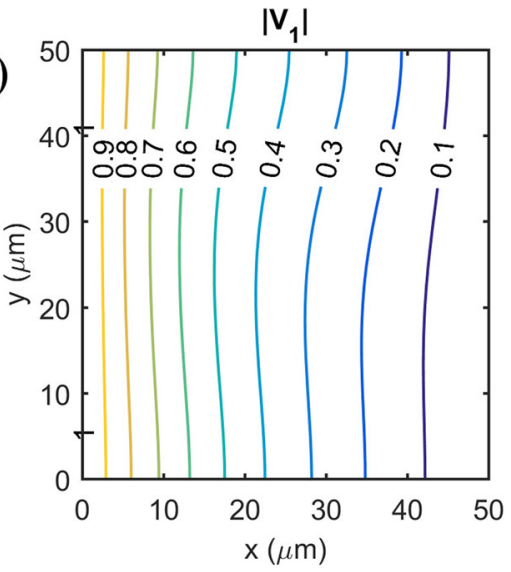

(c)

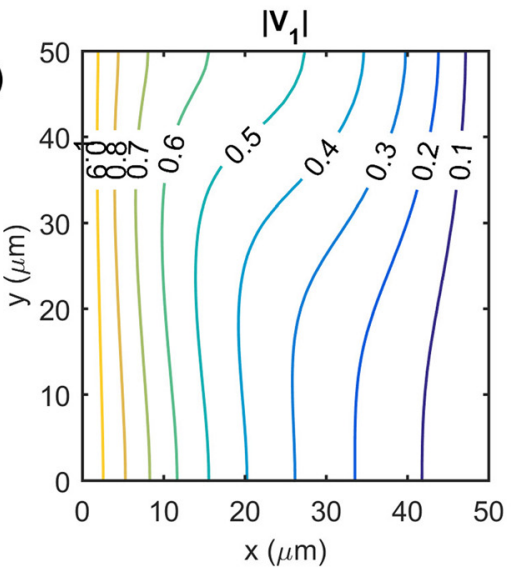

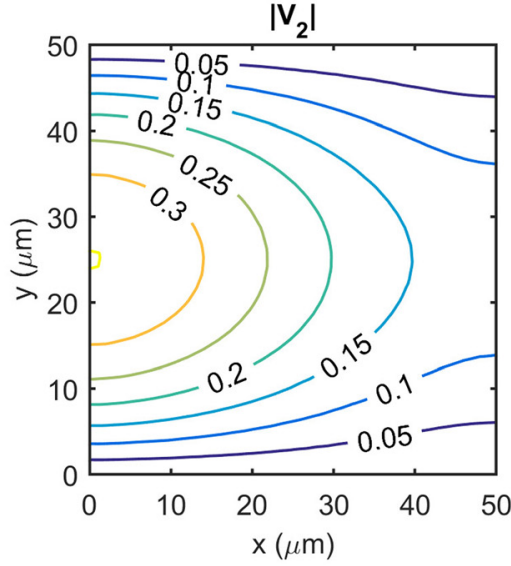
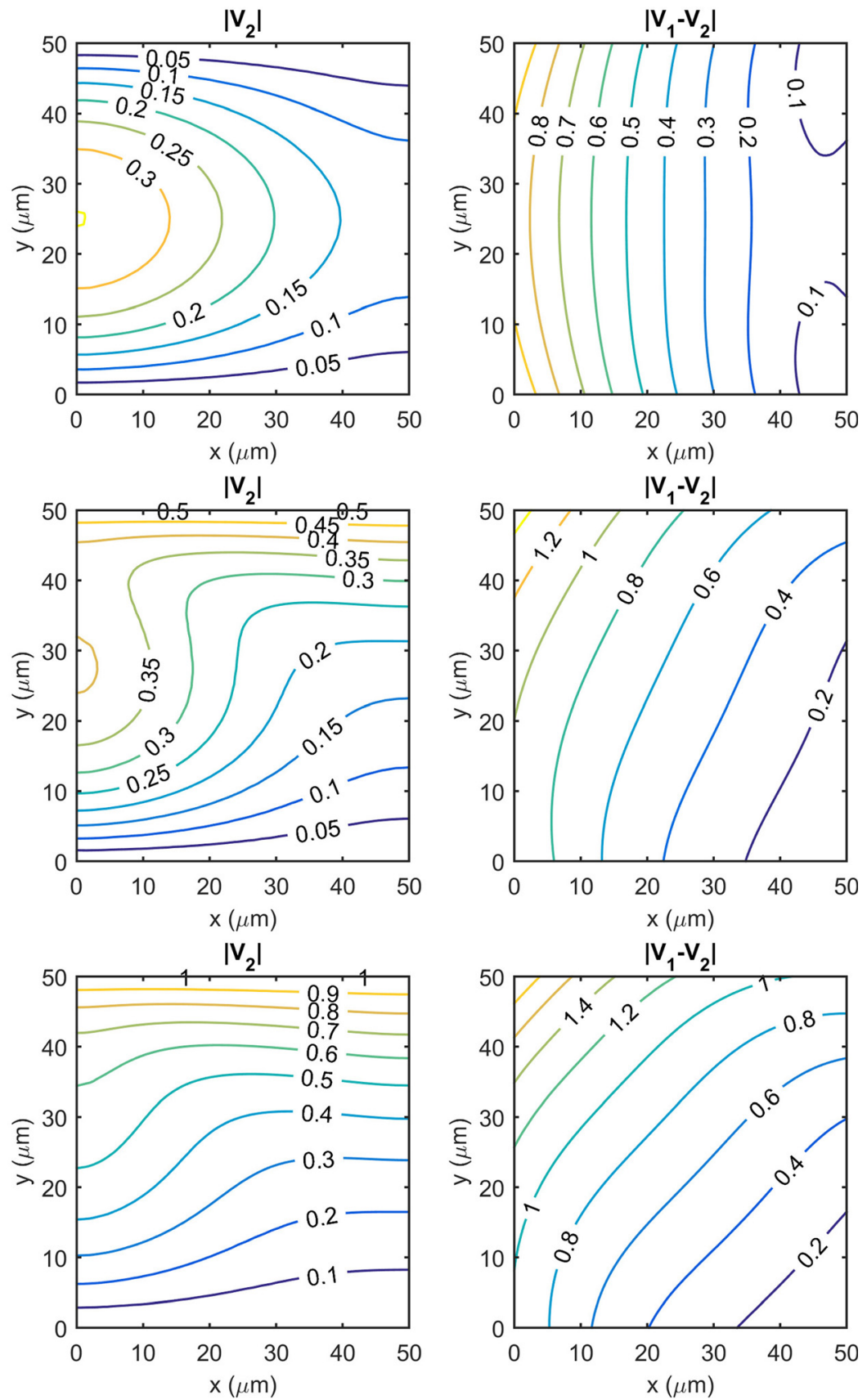

FIG. 13. Absolute value of the potential distribution at the top and bottom (left and middle figures) and potential difference between top and bottom (right) figures for a configuration for two-dimensional beam steering. (a) $V_{A}=1 \mathrm{~V}, V_{B}=V_{C}=V_{D}=0 \mathrm{~V}$; (b) $V_{A}=1 \mathrm{~V}, V_{B}=V_{C}=0 \mathrm{~V}, V_{D}=0.5 \mathrm{~V}$; (c) $V_{A}=V_{D}=1 \mathrm{~V}$, $V_{B}=V_{C}=0 \mathrm{~V}$. 
parabolic profile. The results for the focal distance and the aberration (i.e., coefficient $a_{8}$ given in a number of wavelengths) are shown in Fig. 11. Of course, also higher order aberrations are occurring but are not discussed here. For both characteristic lengths, there is a voltage region in which the focal distance variation is fairly linear with applied voltage. Also in this voltage region, the lowest lens aberrations can be found. The two figures clearly demonstrate the trade-off which needs to be made in terms of characteristic length. Higher conductivity (smaller characteristic length) leads to lower overall aberration, but gives rises to a longer minimal focal distance.

\section{E. Two-dimensional steering with two weakly conductive layers}

As an example of a configuration with weakly conductive layers on both sides of the LC layer, we investigate whether two-dimensional steering is possible with two straight Perfect Electric Conductors (PECs) at the bottom along the $y$ direction and two straight PECs at the top along the $x$ direction as shown in Fig. 12. Both top and bottom PECs are $50 \mu \mathrm{m}$ apart from each other, while $\sigma_{1} d_{1}=\sigma_{2} d_{2}$. The contour plots of the potential distribution for 3 different applied voltages are shown in Fig. 13. When examining the potential difference (figures on the right side), it is clear that a fairly linear voltage profile can be realized along any direction in the $x y$-plane, demonstrating the possibility of twodimensional steering. However, the situation is different from the one-dimensional steering case. As it is clear from Fig. 6, in the one-dimensional case, it is possible to extend the configuration periodically such that large blazed gratings can be realized. In the two-dimensional case, in general, this is not possible anymore. The steering is then limited to a one-period device. Nevertheless we believe that even a oneperiod device has important applications in which for example, a focused laser beam can be steered in any possible direction. In such an application, the laser beam must be focused such that its waist is smaller than the region formed by the four contacting electrodes. As a result of the focusing, there is a considerable divergence of the beam. It is clear that the angle over which the beam can be steered must be much larger than the beam divergence angle. To the best of our knowledge, we believe that such a device has not been presented yet in the literature.

\section{CONCLUSION}

In this paper, we have developed an approximated quasi-static model for describing LC devices with weakly conductive electrodes driven by sinusoidally varying voltages. The nonlinear partial differential equations are solved using finite element discretization. The model offers an easy way to estimate the LC behavior in such devices by reducing the number of dimensions by one and thus avoiding the need for full three-dimensional calculation of the potential distribution inside the devices. The model is applied to a number of interesting configurations. A novel two-dimensional steering device is proposed and its working principle is demonstrated.

\section{ACKNOWLEDGMENTS}

This work was funded by the IWT SBO-Project SECONDOS and the Interuniversity Attraction Poles program of the Belgian Science Policy Office, under Grant No. IAP P7-35 (Photonics@be). Inge Nys is Ph.D. fellow of the Research Foundation, Flanders.

${ }^{1}$ N. T. Nguyen, Biomicrofluidics 4, 031501 (2010).

${ }^{2}$ B. Berge and J. Peseux, Eur. Phys. J. E 3, 159 (2000).

${ }^{3}$ J. Beeckman, K. Neyts, and P. Vanbrabant, Opt. Eng. 50, 081202 (2011).

${ }^{4}$ S. Sato, Jpn. J. Appl. Phys. 18, 1679 (1979).

${ }^{5}$ H. W. Ren and S. T. Wu, Opt. Express 14, 11292 (2006).

${ }^{6} \mathrm{~K}$. Asatryan, V. Presnyakov, A. Tork, A. Zohrabyan, A. Bagramyan, and

T. Galstian, Opt. Express 18, 13981 (2010).

${ }^{7}$ H. C. Lin and Y. H. Lin, Opt. Express 20, 2045 (2012).

${ }^{8}$ L. W. Li, D. Bryant, T. Van Heugten, and P. J. Bos, Opt. Express 21, 8371 (2013).

${ }^{9}$ P. Valley, D. L. Mathine, M. R. Dodge, J. Schwiegerling, G. Peyman, and N. Peyghambarian, Opt. Lett. 35, 336 (2010).

${ }^{10}$ O. Willekens, J. P. George, K. Neyts, and J. Beeckman, Opt. Express 24, 8088 (2016).

${ }^{11}$ M. Ye, B. Wang, and S. Sato, Opt. Express 16, 4302 (2008).

${ }^{12}$ N. Fraval and J. L. D. de la Tocnaye, Appl. Opt. 49, 2778 (2010).

${ }^{13}$ Y. H. Lin, H. S. Chen, C. M. Chang, and Y. J. Wang, Liq. Cryst. XVIII 9182, 91820B (2014).

${ }^{14}$ N. Fraval, F. Berier, and O. Castany, MOEMS Miniaturized Syst. XI 8252, 82520Q (2012).

${ }^{15}$ W. Klaus, M. Ide, S. Morokawa, M. Tsuchiya, and T. Kamiya, Opt. Commun. 138, 151 (1997).

${ }^{16}$ X. Shang, A. M. Trinidad, P. Joshi, J. D. Smet, D. Cuypers, and H. D. Smet, IEEE Photonics J. 8, 1 (2016).

${ }^{17}$ K. Neyts, A. Real, M. Marescaux, S. Mladenovski, and J. Beeckman, J. Appl. Phys. 103, 093113 (2008).

${ }^{18}$ H. N. Tang, Y. B. Jiang, C. W. Tang, and H. S. Kwok, J. Disp. Technol. 12, 605 (2016).

${ }^{19}$ A. Cheknane, B. Benyoucef, J. P. Charles, R. Zerdoum, and M. Trari, Sol. Energy Mater. Sol. Cells 87, 557 (2005).

${ }^{20} \mathrm{~K}$. Neyts and F. Beunis, "Ion transport in liquid crystals," in Handbook of Liquid Crystals (Wiley-VCH Verlag GmbH \& Co. KGaA, 2014), Chap. 11 .

${ }^{21}$ R. James, F. A. Fernandez, S. E. Day, M. Komarcevic, and W. A. Crossland, Mol. Cryst. Liquid Cryst. 422, 209 (2004).

${ }^{22}$ R. James, E. Willman, F. A. Fernández, and S. E. Day, IEEE Trans. Electron Devices 53, 1575 (2006).

${ }^{23}$ P. G. de Gennes and J. Prost, The Physics of Liquid Crystals, International Series of Monographs on Physics (Oxford University Press, Oxford, 1995).

${ }^{24}$ J. Beeckman, K. Neyts, and M. Haelterman, J. Opt. A: Pure Appl. Opt. 8, 214-220 (2006).

${ }^{25}$ H. Gross, H. Zügge, M. Peschka, and F. Blechinger, "Aberrations," in Handbook of Optical Systems (Wiley-VCH Verlag GmbH \& Co. KGaA, 2006), pp. 1-70. 